\title{
ERRATUM
}

\section{Pharmacogenetic comparison of CYP2D6 predictive and measured phenotypes in a South African cohort}

TM Dodgen, C De J Labuschagne, A van Schalkwyk, FE Steffens, A Gaedigk, AD Cromarty, M Alessandrini and MS Pepper

The Pharmacogenomics Journal (2017) 17, 393; doi:10.1038/tpj.2017.20

Correction to: The Pharmacogenomics Journal (2016) 16, 566-572; doi:10.1038/tpj.2015.76; published online 27 October 2015

In the originally published version of this paper, the author affiliations were incorrect. The correct affiliations appear below. The publisher regrets the error.

TM Dodgen ${ }^{1,2}$, C De J Labuschagne ${ }^{3}$, A van Schalkwyk ${ }^{3}, \mathrm{FE}$ Steffens $^{4}$, A Gaedigk ${ }^{5}$, AD Cromarty ${ }^{1}, M$ Alessandrini ${ }^{2}$ and MS Pepper ${ }^{2,6}$

${ }^{1}$ Department of Pharmacology, University of Pretoria, Pretoria, South Africa;
2Department of Immunology and Institute for Cellular and Molecular Medicine, University of Pretoria, Pretoria, South Africa;

${ }^{3}$ Inqaba Biotechnical Industries, Pretoria, South Africa;

${ }^{4}$ Department of Consumer Science, University of Pretoria, Pretoria, South Africa;

${ }^{5}$ Division of Clinical Pharmacology, Toxicology \& Therapeutic Innovation, Children's Mercy Kansas City, and School of Medicine, University of Missouri-Kansas City, Kansas City, MO, USA;

${ }^{6}$ Department of Genetic Medicine and Development, University of Geneva, Geneva, Switzerland. 